Proyecciones Journal of Mathematics

Vol. 25, No 2, pp. 111-120, August 2006.

Universidad Católica del Norte

Antofagasta - Chile

\title{
STRONG TOPOLOGIES FOR MULTIPLIER CONVERGENT SERIES
}

\author{
CHARLES SWARTZ \\ NEW MEXICO STATE UNIVERSITY, U.S.A.
}

Received: December 2005. Accepted : June 2006

\begin{abstract}
P. Dierolf has shown that there is a strongest locally convex polar topology which has the same subseries (bounded multiplier) convergent series as the weak topology, and I. Tweddle has shown that there is a strongest locally convex topology which has the same subseries convergent series as the weak topology. We establish the analogues of these results for multiplier convergent series if the sequence space of multipliers has the signed weak gliding hump property. We compare our main result with other known Orlicz-Pettis Theorems for multiplier convergent series.
\end{abstract}


The classical Orlicz-Pettis (OP) Theorem for normed linear spaces asserts that a series in a normed linear space which is subseries (ss) convergent in the weak topology is actually ss convergent in the norm topology of the space $([\mathrm{O}],[\mathrm{P}])$. The theorem was generalized to locally convex spaces by McArthur and asserts that a series which is ss convergent in the weak topology is ss convergent in the original or the Mackey topology of the space $([\mathrm{Mc}])$. Dierolf has shown that there is a strongest locally convex polar topology which has the same ss convergent series as the weak topology $([\mathrm{D}])$, and Tweddle has shown that there is a strongest locally convex topology which has the same ss convergent series as the weak topology $([\mathrm{T}])$. A series $\sum x_{j}$ in a Hausdorff locally convex space $(E, \tau)$ is ss convergent iff for every subsequence $\left\{x_{n_{j}}\right\}$ the subseries $\sum x_{n_{j}}$ is $\tau$ convergent; if $I=\left\{n_{j}\right\}$ is a subsequence and $C_{I}$ is the characteristic function of $I$, then the subseries $\sum x_{n_{j}}$ is $\tau$ convergent iff the series $\sum_{j=1}^{\infty} C_{I}(j) x_{j}$ is $\tau$ convergent. If $m_{0}=\operatorname{span}\left\{C_{I}: I \subset N\right\}$, then a series $\sum x_{j}$ in $(E, \tau)$ is ss convergent iff $\sum_{j=1}^{\infty} t_{j} x_{j}$ is $\tau$ convergent for every $t=\left\{t_{j}\right\} \in m_{0}$. This suggests that the sequence space $m_{0}$ could be replaced by other sequence spaces and the resulting series could be studied. If $\lambda$ is any vector space of scalar sequences which contains the space $c_{00}$ of sequences which are eventually 0 , a series $\sum x_{j}$ in $(E, \tau)$ is said to be $\tau \lambda$-multiplier convergent if the series $\sum t_{j} x_{j}$ is $\tau$ convergent for every $t=\left\{t_{j}\right\} \in \lambda$; thus, a series is ss convergent iff the series is $m_{0}$-multiplier convergent. OP Theorems for multiplier convergent series have been established by several authors ([LCC],[WL],[SS1],[SS2],[Sw3]) and, in particular an OP Theorem for locally convex spaces has been established for multiplier spaces $\lambda$ with a property called the signed weak gliding hump property ([SS2]). In this note we show that the analogues for the Dierolf and Tweddle topologies exist for $\lambda$-multiplier convergent series when $\lambda$ has the signed weak gliding hump property. We then show that if the multiplier space $\lambda$ has a gliding hump property, called the signed weak gliding hump property, then the Dierolf topology is stronger than the Mackey topology and 2 other topologies often considered for the OP Theorem.

Let $E, E^{\prime}$ be a dual pair with respect to the bilinear pairing $\langle$,$\rangle . The$ weak (Mackey, strong) topology on $E$ with respect to this duality is denoted by $\sigma\left(E, E^{\prime}\right)\left(\tau\left(E, E^{\prime}\right), \beta\left(E, E^{\prime}\right)\right)([\mathrm{Wi}]$, [Sw1 ] ). Let $\lambda$ be a sequence space containing $c_{00}$. The $\beta$-dual of $\lambda$ is defined to be $\lambda^{\beta}=\left\{\left\{s_{j}\right\}: \sum s_{j} t_{j}\right.$ converges for every $\left.t=\left\{t_{j}\right\} \in \lambda\right\}$; if $t=\left\{t_{j}\right\} \in \lambda$ and $s=\left\{s_{j}\right\} \in \lambda^{\beta}$, we write $s \cdot t=\sum s_{j} t_{j}$ and note that $\lambda, \lambda^{\beta}$ are in duality with respect to the bilinear pairing $s \cdot t$.

The Dierolf topology is the polar topology defined by the following col- 
lection $M$ of $\sigma\left(E^{\prime}, E\right)$ bounded subsets of $E^{\prime}$;

$M \in M$ iff $M$ is $\sigma\left(E^{\prime}, E\right)$ bounded and for every linear continuous operator $S:\left(E^{\prime}, \sigma\left(E^{\prime}, E\right)\right) \rightarrow\left(l^{1}, \sigma\left(l^{1}, m_{0}\right)\right) S M$ is relatively compact in $\left(l^{1},\|\|_{1}\right)$.

The Dierolf topology is the polar topology of uniform convergence on the members of $M$ and is the strongest polar topology with the same ss convergent series as $\sigma\left(E, E^{\prime}\right)([\mathrm{D}])$; see $[\mathrm{K}],[\mathrm{Wi}],[\mathrm{Sw} 1]$ for a discussion of polar topologies.)

The development of the basic properties of the Dierolf topology is based on the following facts concerning $m_{0}$ and its $\beta$-dual $l^{1}$ : Let $K \subset l^{1}$. The following are equivalent:

(i) $K$ is $\sigma\left(l^{1}, m_{0}\right)$ compact

(ii) $K$ is \|\|$_{1}$ compact

(iii) $\lim _{n} \sum_{j=n}^{\infty}\left|t_{j}\right|=0$ uniformly for $t=\left\{t_{j}\right\} \in K$.

(See $[\mathrm{BK}],[\mathrm{K}] 22.4 .(3)$.

For use in our generalization of the Dierolf topology for general spaces of multipliers, we establish another condition equivalent to (i)-(iii).

(iv) for every $t=\left\{t_{i}\right\} \in m_{0}, \lim _{n} \sum_{j=n}^{\infty} t_{j} x_{j}=0$ uniformly for $x \in K$.

That (iii) implies (iv) ( even for $t \in l^{\infty}$ ) is clear. Suppose that (iii) fails. Then there exists $\epsilon>0$ such that for every $k$ there exist $m_{k}>k, x^{k} \in K$ such that $\sum_{i=m_{k}}^{\infty}\left|x_{i}^{k}\right|>5 \epsilon$. In particular, there exist $m_{1}, x^{1} \in K$ such that $\sum_{i=m_{1}}^{\infty}\left|x_{i}^{1}\right|>5 \epsilon$. There exists $n_{1}>m_{1}$ such that $\sum_{i=n_{1}+1}^{\infty}\left|x_{i}^{1}\right|<\epsilon$. Therefore, $\sum_{i=m_{1}}^{n_{1}}\left|x_{i}^{1}\right|>4 \epsilon$. Set $I_{1}=\left[m_{1}, n_{1}\right], I_{1}^{+}=\left\{i \in I_{1}: x_{i}^{1}>\right.$ $0\}, I_{1}^{-}=\left\{i \in I_{1}: x_{i}^{1}<0\right\}$. Either $\left|\sum_{i \in I_{1}^{+}} x_{i}^{1}\right|>2 \epsilon$ or $\left|\sum_{i \in I_{1}^{-}} x_{i}^{1}\right|>2 \epsilon$; pick one of these index sets which gives this inequality and label it $J_{1}$ so $\left|\sum_{i \in J_{1}} x_{i}^{1}\right|>2 \epsilon$. Continuing this construction produces an increasing sequence of subsets $\left\{J_{k}\right\}, x^{k} \in K, m_{k}<n_{k}<m_{k+1}<n_{k+1}<\ldots$ with $J_{k} \subset\left[m_{k}, n_{k}\right],\left|\sum_{i \in J_{k}} x_{i}^{k}\right|>2 \epsilon, \sum_{i=n_{k}+1}^{\infty}\left|x_{i}^{k}\right|<\epsilon$. Put $t=\sum_{i=1}^{\infty} C_{J_{i}}$ (coordinate sum). Then hold.

$\left|\sum_{i=m_{k}}^{\infty} t_{i} x_{i}^{k}\right| \geq\left|\sum_{i \in J_{k}} x_{i}^{k}\right|-\sum_{i=n_{k}+1}^{\infty}\left|x_{i}^{k}\right|>2 \epsilon-\epsilon=\epsilon$ so (iii) fails to

For the case of a general space of multipliers, we make the following definition of a condition analogous to condition (iv).

Definition 1. A subset $K \subset \lambda^{\beta}$ has uniform tails if for every $t \in \lambda$, $\lim _{n} \sum_{j=n}^{\infty} t_{j} s_{j}=0$ uniformly for $s \in K$.

Using the equivalence of conditions (ii) and (iv) above, the analogue of the Dierolf topology for general spaces of multipliers is defined to be the polar topology generated by the family $D=D_{\lambda}$ : 
$D \in D_{\lambda}$ iff $D$ is $\sigma\left(E^{\prime}, E\right)$ bounded and for every linear continuous $S:\left(E^{\prime}, \sigma\left(E^{\prime}, E\right)\right) \rightarrow\left(\lambda^{\beta}, \sigma\left(\lambda^{\beta}, \lambda\right)\right) S D$ has uniform tails in $\lambda^{\beta}$.

As above, we denote the polar topology of uniform convergence on the members of $D_{\lambda}$ by $D_{\lambda}\left(E, E^{\prime}\right)$. It follows from the equivalence of (i)-(iv) above that when $\lambda=m_{0}$ the Dierolf topology is exactly $D_{m_{0}}\left(E, E^{\prime}\right)$ so it is reasonable to view this topology as a generalization of the Dierolf topology.

In order to establish the basic property of the generalized Dierolf topology we prove the following result.

Proposition 2. There is a 1-1 correspondence between $\sigma\left(E, E^{\prime}\right) \lambda$-multiplier convergent series $\sum x_{j}$ and linear continuous operators $S:\left(E^{\prime}, \sigma\left(E^{\prime}, E\right)\right) \rightarrow$ $\left(\lambda^{\beta}, \sigma\left(\lambda^{\beta}, \lambda\right)\right)$. The correspondence is given by $x_{j}=S^{\prime} e^{j}$, where $e^{j}$ is the sequence with 1 in the $j t h$ coordinate and 0 in the other coordinates and $S^{\prime}$ is the adjoint operator.

Proof: Suppose that $\sum x_{j}$ is $\sigma\left(E, E^{\prime}\right) \lambda$-multiplier convergent. Define $T: \lambda \rightarrow E$ by $T t=\sum t_{j} x_{j}\left(\sigma\left(E, E^{\prime}\right)\right.$ sum $)$. We claim that $T$ is $\sigma\left(\lambda, \lambda^{\beta}\right)-$ $\sigma\left(E, E^{\prime}\right)$ continuous. Let $x^{\prime} \in E^{\prime}, t \in \lambda$. Then $\left\langle x^{\prime}, T t\right\rangle=\left\langle x^{\prime}, \sum t_{j} x_{j}\right\rangle=$ $\sum t_{j}\left\langle x^{\prime}, x_{j}\right\rangle=t \cdot\left\{\left\langle x^{\prime}, x_{j}\right\rangle\right\}$ where $\left\{\left\langle x^{\prime}, x_{j}\right\rangle\right\} \in \lambda^{\beta}$. This implies that $T$ is $\sigma\left(\lambda, \lambda^{\beta}\right)-\sigma\left(E, E^{\prime}\right)$ continuous. Therefore $S=T^{\prime}$ is $\sigma\left(E^{\prime}, E\right)-\sigma\left(\lambda^{\beta}, \lambda\right)$ continuous ([Sw1]26.14).

If $S:\left(E^{\prime}, \sigma\left(E^{\prime}, E\right)\right) \rightarrow\left(\lambda^{\beta}, \sigma\left(\lambda^{\beta}, \lambda\right)\right)$ is linear and continuous, then $S^{\prime}=$ $T: \lambda \rightarrow E$ is $\sigma\left(\lambda, \lambda^{\beta}\right)-\sigma\left(E, E^{\prime}\right)$ continuous ([Sw1]26.14). Now $\sum e^{j}$ is $\sigma\left(\lambda, \lambda^{\beta}\right) \lambda$-multiplier convergent so $\sum S^{\prime} e^{j}=\sum T e^{j}=\sum x_{j}$ is $\sigma\left(E, E^{\prime}\right)$ $\lambda$-multiplier convergent and the correspondence follows.

Thus, it follows from Proposition 2, to check that a subset $D \subset E^{\prime}$ belongs to $D_{\lambda}$, it suffices to show that $\lim _{n} \sum_{j=n}^{\infty}\left\langle x^{\prime}, x_{j}\right\rangle=0$ uniformly for $x^{\prime} \in D$ whenever $\sum x_{j}$ is a $\sigma\left(E, E^{\prime}\right) \lambda$-multiplier convergent series.

It follows from Proposition 2 that $D_{\lambda}\left(E, E^{\prime}\right)$ is the strongest polar topology on $E$ with the same $\lambda$-multiplier convergent series as $\sigma\left(E, E^{\prime}\right)$.

Theorem 3. $D_{\lambda}\left(E, E^{\prime}\right)$ is the strongest polar topology on $E$ with the same $\lambda$ multiplier convergent series as $\sigma\left(E, E^{\prime}\right)$.

Proof: Suppose $\sum x_{j}$ is $\sigma\left(E, E^{\prime}\right) \lambda$ multiplier convergent. Let $T: \lambda \rightarrow E$ be defined as in Proposition $2, T^{\prime}=S$ and let $D \in D_{\lambda}$. Then $S D=$ 
$\left\{\left\{\left\langle x^{\prime}, x_{j}\right\rangle\right\}: x^{\prime} \in D\right\}$ has uniform tails in $\lambda^{\beta}$ so if $t \in \lambda, \lim _{n} \sum_{j=n}^{\infty} t_{j}\left\langle x^{\prime}, x_{j}\right\rangle=$ 0 uniformly for $x^{\prime} \in D$. That is, $\sum t_{j} x_{j}$ is $D_{\lambda}\left(E, E^{\prime}\right)$ convergent.

Suppose that $\alpha$ is a polar topology on $E$ with the same $\lambda$ multiplier convergent series as $\sigma\left(E, E^{\prime}\right)$. If $\alpha$ is the polar topology of uniform convergence on members of $A$ and if $\sum x_{j}$ is $\sigma\left(E, E^{\prime}\right) \lambda$ convergent, $t \in \lambda$ and $A \in A$, then $\lim _{n} \sum_{j=n}^{\infty} t_{j}\left\langle x^{\prime}, x_{j}\right\rangle=0$ uniformly for $x^{\prime} \in A$. Hence, $A \in D_{\lambda}$ and $\alpha$ is weaker than $D_{\lambda}\left(E, E^{\prime}\right)$.

It is known in the locally convex case that there are locally convex topologies which are stronger than the Mackey topology and have the same ss convergent series as $\sigma\left(E, E^{\prime}\right)$. We now compare the Dierolf topology with 2 of these locally convex topologies.

For this we next establish a lemma which will be used several times in what follows. A sequence of intervals $\left\{I_{j}\right\}$ in $N$ is increasing if $\max I_{j}<$ $\min I_{j+1}$.

Lemma 4. Suppose $M \subset E^{\prime}$ is such that there exist a $\sigma\left(E, E^{\prime}\right) \lambda$-multiplier convergent series $\sum x_{j}$ and $t \in \lambda$ such that the series $\sum t_{j}\left\langle x^{\prime}, x_{j}\right\rangle$ do not converge uniformly for $x^{\prime} \in M$. Then there exist $\epsilon>0,\left\{x_{k}^{\prime}\right\} \subset M$ and an increasing sequence of intervals $\left\{I_{k}\right\}$ with $\left|\sum_{j \in I_{k}} t_{j}\left\langle x_{k}^{\prime}, x_{j}\right\rangle\right|>\epsilon$ for every $k$.

Proof: If the series do not converge uniformly, then there exists $\epsilon>0$ such that for every $k$ there exist $m_{k}>k, x^{\prime}=x^{\prime}(k) \in M$ such that $\left|\sum_{j=m_{k}}^{\infty} t_{j}\left\langle x^{\prime}, x_{j}\right\rangle\right|>2 \epsilon$. In particular, there exist $m_{1}, x_{1}^{\prime} \in M$ with $\left|\sum_{j=m_{1}}^{\infty} t_{j}\left\langle x_{1}^{\prime}, x_{j}\right\rangle\right|>2 \epsilon$. Since $\sum x_{j}$ is $\sigma\left(E, E^{\prime}\right) \lambda$-multiplier convergent, there exists $n_{1}>m_{1}$ such that $\left|\sum_{j=n_{1}+1}^{\infty} t_{j}\left\langle x_{1}^{\prime}, x_{j}\right\rangle\right|<\epsilon$. Thus, if $I_{1}=\left[m_{1}, n_{1}\right]$, then $\left|\sum_{j \in I_{1}} t_{j}\left\langle x_{1}^{\prime}, x_{j}\right\rangle\right|>\epsilon$. Continuing this construction establishes the lemma.

In order to establish our results we impose a gliding hump property on the multiplier space. The space $\lambda$ has the signed weak gliding hump property (signed WGHP) if for every $t \in \lambda$ and every increasing sequence of intervals $\left\{I_{j}\right\}$, there is a subsequence $\left\{n_{j}\right\}$ and a sequence of signs $\epsilon_{j}= \pm 1$ such that the coordinatewise sum of the series $\sum_{j=1}^{\infty} \epsilon_{j} C_{I_{n_{j}}} t$ belongs to $\lambda$; if the signs $\epsilon_{j}$ can all be chosen to be equal to 1 , then $\lambda$ is said to have the weak gliding hump property (WGHP). For example, any monotone space such as $c_{00}, c_{0}, m_{0}$, or $l^{p}(0<p \leq \infty)$ has WGHP whereas the non-monotone space $b s$, the space of bounded series, has signed WGHP but not WGHP (see 
[SS1],[SS2] or [Sw2] for further examples and applications; [BSS] contains many examples of non-monotone spaces with signed WGHP).

First, consider the polar topology $\gamma\left(E, E^{\prime}\right)$ of uniform convergence on conditionally $\sigma\left(E^{\prime}, E\right)$ sequentially compact subsets of $E^{\prime}$ (i.e., subsets $K \subset$ $E^{\prime}$ such that every sequence $\left\{x_{j}^{\prime}\right\} \subset K$ has a subsequence $\left\{x_{n_{j}}^{\prime}\right\}$ such that $\lim \left\langle x_{n_{j}}^{\prime}, x\right\rangle$ exists for every $x \in E \quad$ (see [D]2.4(e) or [Di]). It has been shown that every $\sigma\left(E, E^{\prime}\right)$ ss convergent series is also $\gamma\left(E, E^{\prime}\right)$ ss convergent ([Sw2]10.3.1, [SS2]).

Theorem 5. Let $\lambda$ have signed WGHP. Then $\gamma\left(E, E^{\prime}\right) \subset D_{\lambda}\left(E, E^{\prime}\right)$.

Proof: Suppose that $K \subset E^{\prime}$ is conditionally $\sigma\left(E^{\prime}, E\right)$ sequentially compact. If $K$ doesn't belong to $D_{\lambda}$, then by Lemma 4 there exist $\epsilon>0,\left\{x_{k}^{\prime}\right\} \subset$ $K$ and an increasing sequence of intervals $\left\{I_{k}\right\}$ with

$$
(*)\left|\sum_{j \in I_{k}} t_{j}\left\langle x_{k}^{\prime}, x_{j}\right\rangle\right|>\epsilon
$$

for every $k$. By the conditional $\sigma\left(E^{\prime}, E\right)$ sequential compactness we may assume that $\lim \left\langle x_{k}^{\prime}, x\right\rangle$ exists for every $x \in E$. Consider the matrix $M=$ $\left[m_{i j}\right]=\left[\sum_{l \in I_{i}} t_{l}\left\langle x_{j}^{\prime}, x_{l}\right\rangle\right]$. We show that $M$ is a signed $K$-matrix in the sense of Antosik and Mikusinski so the diagonal of $M$ should converge to 0 but this will contradict $(*)$ (see [S1],[S2] or [Sw2]2.2.2). First, the columns of $M$ converge by the compactness. Next, given any subsequence there is a further subsequence $\left\{p_{j}\right\}$ and a sequence of signs $\epsilon_{j}$ such that $s=\left\{s_{j}\right\}=\sum_{j=1}^{\infty} \epsilon_{j} C_{I_{p_{j}}} t \in \lambda$. Then $\sum_{j=1}^{\infty} \epsilon_{j} m_{i p_{j}}=\left\langle x_{k}^{\prime}, \sum_{j=1}^{\infty} s_{j} x_{j}\right\rangle \rightarrow 0$. Hence, $M$ is a signed $K$-matrix as claimed and the result follows.

Next, consider the polar topology $\lambda\left(E, E^{\prime}\right)$ of uniform convergence on $\sigma\left(E^{\prime}, E\right)$ compact subsets of $E^{\prime}$. This topology is obviously stronger than the Mackey topology and can be strictly stronger ([D]3.1). The topologies $\lambda\left(E, E^{\prime}\right)$ and $\sigma\left(E, E^{\prime}\right)$ have the same ss convergent series ([BK]2.1,[D]).

Theorem 6. Let $\lambda$ have signed WGHP. Then $\lambda\left(E, E^{\prime}\right) \subset D_{\lambda}\left(E, E^{\prime}\right)$.

Proof:Let $B \subset E^{\prime}$ be $\sigma\left(E^{\prime}, E\right)$ compact. Assume that $B$ doesn't belong to $D_{\lambda}$ and let the notation be as in the proof of Theorem 4 . Let $E_{0}=\operatorname{span}\left\{x_{j}\right\}$. The set $\left\{x_{k}^{\prime}: k\right\}$ is relatively $\sigma\left(E_{0}^{\prime}, E_{0}\right)$ compact and, therefore, relatively $\sigma\left(E_{0}^{\prime}, E_{0}\right)$ sequentially compact since $E_{0}$ is separable ([Wi] $\left.] .5 .3\right)$. Therefore, 
we may assume that $\lim \left\langle x_{k}^{\prime}, x\right\rangle$ exists for every $x \in E_{0}$. The proof can now be completed as in Theorem 5 .

It follows from Theorems 5 and 6 that if $\lambda$ has the signed WGHP, then any series which is $\sigma\left(E, E^{\prime}\right) \lambda$-multiplier convergent is also $\gamma\left(E, E^{\prime}\right), \lambda\left(E, E^{\prime}\right)$ and $\tau\left(E, E^{\prime}\right) \lambda$-multiplier convergent. This result covers series which are not ss convergent and not covered by Dierolf's result ; for example, $b s$-multiplier convergent series may not be subseries convergent since $b s$ is not a monotone space (other examples of non-monotone spaces with signed WGHP are given in [BSS]). See Example 6 of [SS2] for a specific example of a $b s$-multiplier convergent series which is not ss convergent.

Without some assumptions on the multiplier space $\lambda$, the inclusions in Theorems 5 and 6 may not hold.

Example 7. Let $\mu$ be the space of all scalar sequences which are eventually constant. Then if $\tau$ is any locally convex topology on $E$, a series $\sum x_{j}$ in $E$ is $\tau \mu$-multiplier convergent iff $\sum x_{j}$ is $\tau$ convergent. Thus, if one chooses a series which is $\sigma\left(E, E^{\prime}\right)$ convergent but not $\tau\left(E, E^{\prime}\right)$, the series is $\sigma\left(E, E^{\prime}\right) \mu$-multiplier convergent but not $\tau\left(E, E^{\prime}\right) \mu$-multiplier convergent. [For example, $\sum\left(e^{k+1}-e^{k}\right)$ is $\sigma\left(c_{0}, l^{1}\right)$ convergent but not \|\|$_{\infty}$ convergent.]

Tweddle has shown that there is a strongest locally convex topology, $t\left(E, E^{\prime}\right)$, on $E$ which has the same ss convergent series as $\sigma\left(E, E^{\prime}\right)([T])$. We now establish the analogue of Tweddle's result for multiplier convergent series. Let $G^{\prime}$ be the space of all linear functionals $x^{\prime}$ on $E$ such that $\sum t_{i}\left\langle x^{\prime}, x_{i}\right\rangle=\left\langle x^{\prime}, \sum t_{i} x_{i}\right\rangle$ for every $\sigma\left(E, E^{\prime}\right) \lambda$-multiplier convergent series $\sum x_{i}$ and for every $t \in \lambda$.

Theorem 8. Let $\lambda$ have signed WGHP. Then $D_{\lambda}\left(E, G^{\prime}\right)$ is the strongest locally convex topology on $E$ which has the same $\lambda$-multiplier convergent series as $\sigma\left(E, E^{\prime}\right)$.

Proof: If $\sum x_{i}$ is $\sigma\left(E, E^{\prime}\right) \lambda$-multiplier convergent, then $\sum x_{i}$ is $\sigma\left(E, G^{\prime}\right)$ $\lambda$-multiplier convergent and ,therefore, $D_{\lambda}\left(E, G^{\prime}\right) \lambda$-multiplier convergent .

Suppose that $\nu$ is a locally convex topology on $E$ with the same $\lambda$ multiplier convergent series as $\sigma\left(E, E^{\prime}\right)$.Put $H^{\prime}=(E, \nu)^{\prime}$. Then $H^{\prime} \subset G^{\prime}$ so by Theorem 6

$$
\nu \subset \tau\left(E, H^{\prime}\right) \subset D_{\lambda}\left(E, H^{\prime}\right) \subset D_{\lambda}\left(E, G^{\prime}\right) .
$$


In general, a series which is ss convergent in the weak topology $\sigma\left(E, E^{\prime}\right)$ is not ss convergent in the strong topology $\beta\left(E, E^{\prime}\right)\left[\right.$ consider $\sum e^{k}$ in $\sigma\left(l^{\infty}, l^{1}\right)$ where $\left.\beta\left(l^{\infty}, l^{1}\right)=\|\|_{\infty}\right]$. However, if the multiplier space $\lambda$ satisfies sufficiently strong conditions, it is known that a $\sigma\left(E, E^{\prime}\right)$ multiplier convergent series will be $\beta\left(E, E^{\prime}\right)$ multiplier convergent ([LCC],[Sw3]). We show that if the multiplier space $\lambda$ satisfies the gliding hump property of [Sw3], then the strong topology is weaker than $D_{\lambda}\left(E, E^{\prime}\right)$ so any $\sigma\left(E, E^{\prime}\right) \lambda$-multiplier convergent series is also $\beta\left(E, E^{\prime}\right) \lambda$-multiplier convergent.

Definition 9. The multiplier space $\lambda$ has the infinite gliding hump property $(\infty-\mathrm{GHP})$ if whenever $t \in \lambda$ and $\left\{I_{k}\right\}$ is an increasing sequence of intervals, there exist a subsequence $\left\{n_{k}\right\}$ and $a_{n_{k}}>0, a_{n_{k}} \rightarrow \infty$ such that every subsequence of $\left\{n_{k}\right\}$ has a further subsequence $\left\{p_{k}\right\}$ such that $\sum_{k=1}^{\infty} a_{p_{k}} C_{I_{p_{k}}} t \in \lambda$ (coordinate sum). [The term $\infty$-GHP is used to suggest that the "humps" $C_{I_{k}} t$ are multiplied by terms in a sequence which tends to $\infty$.]

A list of spaces, some of which are non-monotone, and which satisfy $\infty$-GHP is given in [Sw3] ( for example, $l^{p}(0<p<\infty), c_{0}, c s$ ), where it is shown that if $\lambda$ has $\infty$-GHP, then $\sigma\left(E, E^{\prime}\right)$ and $\beta\left(E, E^{\prime}\right)$ have the same $\lambda$-multiplier convergent series. We now show that if $\lambda$ has $\infty$-GHP , then $\beta\left(E, E^{\prime}\right)$ is weaker than $D_{\lambda}\left(E, E^{\prime}\right)$ so any $\sigma\left(E, E^{\prime}\right) \lambda$-multiplier convergent series is $\beta\left(E, E^{\prime}\right) \lambda$-multiplier convergent recovering one of the main result of $[\mathrm{Sw} 3]$.

Theorem 10. Let $\lambda$ have $\infty$-GHP. Then $\beta\left(E, E^{\prime}\right)$ is weaker than $D_{\lambda}\left(E, E^{\prime}\right)$.

Proof: Let $B \subset E^{\prime}$ be $\sigma\left(E^{\prime}, E\right)$ bounded. Assume that $B$ doesn't belong to $D_{\lambda}$ and let the notation be as in the proof of Theorem 5 . So

$$
\text { (*) }\left|\sum_{l \in I_{k}} t_{l}\left\langle x_{k}^{\prime}, x_{l}\right\rangle\right|>\epsilon
$$

for every $k$ with $x_{k}^{\prime} \in B$. By the $\infty$-GHP, there exist $\left\{p_{k}\right\}, a_{p_{k}}>0, a_{p_{k}} \rightarrow \infty$ such that every subsequence of $\left\{p_{k}\right\}$ has a further subsequence $\left\{q_{k}\right\}$ such that $s=\left\{s_{j}\right\}=\sum_{k} a_{q_{k}} C_{I_{q_{k}}} t \in \lambda$. Consider the matrix $M=\left[m_{i j}\right]=$ $\left[\sum_{l \in I_{j}} t_{l} a_{p_{j}}\left\langle x_{i}^{\prime} / a_{p_{i}}, x_{l}\right\rangle\right]$. We show that $M$ is a $K$-matrix ([Sw2]2.2). Since $x_{i}^{\prime} / a_{p_{i}} \rightarrow 0$ in $\sigma\left(E^{\prime}, E\right)$, the columns of $M$ converge to 0 . Given a subsequence of $\left\{p_{j}\right\}$, let $\left\{q_{j}\right\}$ be a further subsequence as above. Then $\sum_{j=1}^{\infty} m_{i q_{j}}=$ $\sum_{j=1}^{\infty} \sum_{l \in I_{j}} s_{l}\left\langle x_{i}^{\prime} / a_{p_{i}}, x_{l}\right\rangle=\left\langle x_{i}^{\prime} / a_{p_{i}}, \sum_{l} s_{l} x_{l}\right\rangle \rightarrow 0$ where $\sum_{l} s_{l} x_{l}$ is the $\sigma\left(E, E^{\prime}\right)$ sum of the series. Thus, $M$ is a $K$-matrix and the diagonal of $M$ should converge to 0 ([Sw2]2.2.2). But, this contradicts $\left(^{*}\right)$. 


\section{References}

[1] G. Bennett and N. Kalton, FK Spaces containing $c_{0}$, Duke Math. J.,39(1972),561-582.

[2] J. Boos, C. Stuart and C. Swartz,Gliding Hump Properties for Matrix Domains, Analysis Mathematica, 30, pp. 243-257, (2004).

[3] P. Dierolf, Theorems of Orlicz-Pettis type for locally convex spaces, Man. Math., 20, pp. 73-94, (1977).

[4] N. Dinculeanu, Weak compactness and uniform convergence of operators in spaces of Bochner integrable functions, J. Math. Anal. Appl., 109, pp. 372-387, (1985).

[5] G. Kothe, Topological Vector Spaces I, Springer-Verlag, NY, (1983).

[6] Li Ronglu, Cui Chengri and Mingyung Cho, Invariants on all admissible polar topologies, Chinese Annals of Math., 19, pp. 1-6, (1998).

[7] C. McArthur, On a Theorem of Orlicz-Pettis, Pacific J. Math., 22, pp. 297-302, (1967).

[8] W. Orlicz, Bietrage zur Theorie Orthogonalent Wicklungen III, Studia Math., 1, pp. 241-255, (1929).

[9] B. J. Pettis, Integration in Vector Spaces, Trans. Amer. Math. Soc., 44, pp. 277-304, (1938).

[10] C. Stuart, Weak Sequential Completeness in Sequence Spaces, Ph.D. Dissertation, New Mexico State University, (1993).

[11] C. Stuart, Weak Sequential Completeness of $\beta$-Duals, Rocky Mountain Math. J., 26, pp. 1559-1568, (1996).

[12] C. Stuart and C. Swartz, Orlicz-Pettis Theorems for Multiplier Convergent Series, Journal for Analysis and Appl., 17, pp. 805-811, (1998).

[13] C. Stuart and C. Swartz, Generalizations of the Orlicz-Pettis Theorem,

[14] C. Swartz, An Introduction to Functional Analysis, Marcel Dekker, NY, (1992). 
[15] C. Swartz, Infinite Matrices and the Gliding Hump, World Sci. Publ.,Singapore, (1996).

[16] C. Swartz, Orlicz-Pettis Theorems for Multiplier Convergent Operator Valued Series, Proy. J. Math., 23, pp. 61-72, (2004).

[17] I. Tweddle, Vector-Valued Measures, Proc. London Math. Soc., 20, pp. 469-489, (1970).

[18] A. Wilansky, Modern Methods in Topological Vector Spaces, McGrawHill, NY, (1978).

[19] Wu Junde and Li Ronglu, Basic Properties of locally convex A-Spaces, Studia Sci. Math., 35, pp. 353-358, (1999).

\section{Charles Swartz}

Department of Mathematical Sciences

New Mexico State University

Las Cruces

NM 88003

U. S. A.

e-mail : cswartz@nmsu.edu 